\title{
Les espaces de la Russie soviétique vus par Olivier Rolin : entre stéréotype et réalité
}

\author{
The Spaces of the Soviet Russia as Seen by Olivier Rolin: Between \\ Stereotype and Reality
}

\section{RÉSUMÉ}

Dans les années 1980, l'Union soviétique reste encore un pays fermé où les déplacements des touristes internationaux étaient généralement mis sous contrôle. Cependant, Olivier Rolin, écrivain français dont l'oeuvre est inspirée par ses nombreux voyages, parvient à traverser en solitaire ce pays dont il ignorait à peu près tout. Il relate cette "promenade poétique » (comme il qualifie lui-même ce voyage) dans son ouvrage En Russie paru en 1987 et qui englobe les représentations des espaces, des paysages et des modes de vie à travers l'U.R.S.S., notamment les villes soviétiques comme Moscou, Léningrad, Odessa, Yalta, Sotchi, Batoumi, Irkoutsk et Khabarovsk. Ces représentations sont d'ailleurs considérablement influencées par la mythification de la Russie et de l'U.R.S.S. et par des images stéréotypées du pays. Le but de cet article est, ainsi, d'analyser les moyens linguistiques de traduire les stéréotypes et les références culturelles (russes et européennes), dont certains servent d'un appui important à l'auteur cherchant à maîtriser cette altérité qui s'ouvre devant lui, alors que d'autres se voient déconstruits, pour la plupart, face à la réalité.

Mots-clés : représentation linguistique, altérité, stéréotype, U.R.S.S., Olivier Rolin

\section{ABSTRACT}

In the 1980s, the Soviet Union still remained a closed country where the movement of international tourists was generally put under control. However, Olivier Rolin, a French writer whose works are inspired by his many travels, manages to cross alone this country of which he knew almost nothing. He relates this «poetic walk» (as he himself calls it) in his book In Russia published in 1987, which encompasses representations of spaces, landscapes and lifestyles throughout the USSR, including Soviet cities such as Moscow, Leningrad, Odessa, Yalta, Sochi, Batumi, Irkutsk and Khabarovsk. These representations are also significantly influenced by the mythification of Russia and the USSR, and by stereotypical images of the country. The aim of this article is to analyse the linguistic means of translating stereotypes and cultural references (Russian and European ones), some of which serve as an important support to the author seeking to master this otherness that opens to him, while others are deconstructed, for the most part, in front of reality.

Keywords: figurative language, otherness, stereotypes, Soviet Union, Russia, Olivier Rolin

Olga Kulagina, Department of Romance Languages, Moskovskij pedagogicheskij gosudarstvennyj universitet, ul. Malaja Pirogovskaja 1-1, Rossija, Moskva, lynxik@yandex.ru,

https://orcid.org/0000-0002-7382-4751 


\section{Introduction}

La Russie, y compris la Russie soviétique a toujours été l'objet d'une grande curiosité des voyageurs étrangers. En particulier, l'U.R.S.S. d'avant-guerre était souvent vue (notamment par la gauche française) comme le symbole d'un bel avenir heureux, de la liberté et de la véritable émancipation de tous (Garrec, 2011, p. 37), ce qui lui valait une immense popularité parmi les hommes de lettres français : ainsi, le jeune État soviétique a accueilli Romain Rolland, André Gide, Louis Aragon, Elsa Triolet, Louis Guilloux et bien d'autres, l'intérêt des écrivains français pour la Russie et l'U.R.S.S. ayant toujours été et restant encore bien manifeste (la preuve en sont les écrits respectifs du Marquis de Custine, Théophile Gautier, Alexandre Dumas père, Blaise Cendrars, André Gide, Simone de Beauvoir, Olivier Rolin, Sylvain Tesson, Frédéric Beigbeder, Emmanuel Carrère et bien d'autres). Après la Seconde guerre mondiale et jusqu'à la chute de l'U.R.S.S., les relations franco-soviétiques restent plutôt complexes, en passant par des périodes d'entente mais aussi celles de tensions politiques (Mendras, 1985). Toutefois, le tourisme international reprend peu à peu en U.R.S.S., même si le pays reste assez fermé : de ce fait, les touristes ne peuvent se déplacer que sous l'œil vigilant d'un guide et n'ont le droit de voir que ce qui est permis de voir, à savoir les réalisations du régime et de l'idéologie soviétiques (Goldman, 2019). Il en reste de même dans les années 1980, malgré un certain assouplissement du régime qui semblait déjà s'approcher de sa fin. Cependant, Olivier Rolin, écrivain et journaliste français, parvient à traverser en solitaire ce pays dont il ignorait à peu près tout, ce qui s'inscrit d'ailleurs dans sa tendance générale des «parcours solitaires » afin de mieux explorer l'identité collective (Lamarre, 2014, p. 26). C'est dans son récit de voyage En Russie (1987) qu'il relate les détails de cette " promenade poétique » (Rolin, 1997, p. V). C'est sur ce texte que nous nous pencherons afin d'étudier la manière dont l'auteur expose ses impressions, notamment en ce qui concerne la représentation linguistique des espaces et des lieux, dont les espaces urbains, les espaces naturels et les locaux. Les thèses principales que nous avancerons et défendrons dans notre article, sont les suivantes :

1) la perception des espaces soviétiques par l'auteur est considérablement influencée par les stéréotypes;

2) ces stéréotypes sont fondés, pour la plupart, sur les références internationales familières au lectorat français, mais aussi sur les réalités russes et soviétiques qui constituent le bagage culturel de l'auteur et dont beaucoup sont censées être connues en France et en Europe;

3) certains de ces stéréotypes se déconstruisent, face à l'altérité culturelle que l'auteur rencontre au cours de son voyage.

Les méthodes que nous privilégierons sont l'analyse linguistique et l'explication du contexte historique et culturel du texte. 


\section{La grandeur stéréotypée et incommodante}

Il est à noter que la place du stéréotype ${ }^{1}$ est importante dans la vision de la Russie soviétique par l'auteur. Dans l'avertissement à l'édition de 1997, nous voyons une définition géographique suivante de l'U.R.S.S. :

C'était il y a juste dix ans, et il semble qu'un siècle ait passé. Le drapeau rouge flottait encore de l'Elbe au détroit de Béring, l'armée de la même couleur faisait encore trembler l'Europe, Lénine foudroyait de ses millions de regards de bronze ou de ciment, la moindre place de village sur un sixième des terres émergées (Rolin, 1997, p. I).

Dans l'exemple cité ci-dessus, nous sommes en présence de plusieurs hyperboles qui traduisent la vision stéréotypée de l'auteur, dont l'une ( «le drapeau rouge flottait encore de l'Elbe au détroit de Béring ») sert à exagérer la surface de l'Union soviétique qui, en réalité, ne s'est jamais étendue jusqu'à l'Elbe (à moins que l'auteur n'ait en vue les pays du bloc de l'Est qui étaient sous le contrôle plus ou moins explicite de l'U.R.S.S.). Nous rencontrons, dans ce même exemple, deux personnifications hyperboliques, à savoir « l'armée de la même couleur faisait encore trembler l'Europe » et « Lénine foudroyait de ses millions de regards de bronze ou de ciment, la moindre place de village sur un sixième des terres émergées ", qui semblent appuyer, par la mention des omniprésents monuments à Lénine et des ambitions militaires de l'U.R.S.S., la stéréotypisation de l'image du pays.

Dans l'ensemble du texte, l'auteur ne manque pas de mettre en valeur l'étendue importante des espaces en Russie. Toutefois, cette étendue se révèle souvent assez mal organisée, voire inutile. Voici les premières impressions de l'auteur sur Léningrad (qui est aujourd'hui Saint-Pétersbourg) vue par le hublot de l'avion :

Les réacteurs de l'Ilyouchine en remettent dans l'aigu, sous les nuages apparaissent de grands champs pâles, d'autres tirant sur le violet, des bois sombres, pins et bouleaux, des pistes sableuses, des routes rectilignes, étroites, sur lesquelles s'allument les premiers phares. Devant, une côte marécageuse, des îles basses, des lueurs d'eau dans la terre. Alors, c'est ça, la Russie, cette géométrie confuse, ces couleurs mal assurées [...] (p. 17).

Dans cet exemple, l'étendue spatiale des territoires qui s'ouvrent devant l'auteur, est traduite par l'épithète assez explicite « de grands champs », de même que par les énumérations « des bois sombres, pins et bouleaux, des pistes sableuses, des routes rectilignes, étroites, sur lesquelles s'allument les premiers phares " et « une côte marécageuse, des îles basses, des lueurs d'eau dans la terre » qui évoquent la diversité de paysages que l'on découvre à première vue. Cependant,

1 Nous nous référons, dans ce contexte, à la formule de Ruth Amossy qui définit le stéréotype comme un «schème récurrent et figé en prise sur les modèles culturels et les croyances d'une société donnée » (Amossy, 1989, p. 36). 
cette diversité (stéréotype elle-même, en parlant de la Russie) ne semble pas impressionner l'auteur qui exprime, dans le même exemple, sa déception par le biais des épithètes à connotation plutôt négative « cette géométrie confuse, ces couleurs mal assurées »).

L'effet produit sur l'auteur par sa chambre d'hôtel à Léningrad, est à peu près pareil. En voici la preuve : «J'occupe, à moi tout seul, une suite immense, entrée, salon et chambre tendus d'un tissu damassé cramoisi, encombrés d'une quantité déraisonnable de lustres, fauteuils, tables, consoles, coiffeuses et commodes marquetées, rehaussées de bronze doré » (p. 19). Nous sommes en présence, tout d'abord, de l'épithète à valeur hyperbolique " une suite immense » qui traduit la dimension excessive de cette chambre prévue, pourtant, à l'usage d'une seule personne. L'énumération " lustres, fauteuils, tables, consoles, coiffeuses et commodes » (le tout au pluriel, ce qui renforce l'effet de disproportion), ainsi que l'épithète « une quantité déraisonnable », renforce l'effet de l'absurdité de ce faste apparent dont la seule raison d'être consiste à produire l'impression sur le touriste étranger, sans tenir compte de son confort et de l'aspect pratique du logement. Nous noterons également la mention de la couleur cramoisie qui était réservée aux prétendus objets de luxe en U.R.S.S. et qui complète la description de ce décor « de façade ». D'ailleurs, cette mention du cramoisi en tant que symbole d'un luxe douteux n'est pas la seule dans le texte de Rolin, car nous en rencontrons une autre, dans la description d'un hôtel à Odessa :

La salle à manger de l'hôtel Rouge est toute drapée de velours cramoisi. Lustres, colonnes de marbre blanc, plafond à caissons multicolores, en font un décor d'un luxe un peu vulgaire, façon Pompéi revue par Hollywood, où on imagine volontiers Benia Krik, le roi des bandits de l'Odessa babélienne, festoyant avec ses hommes au retour d'un coup (p. 49).

La combinaison du nom de l'hôtel (Krasnaïa en russe, [Rouge] en français) et de la gamme de son décor paraît être une véritable épreuve pour la vue humaine, tout en restant une synthèse du faste excessif et d'une allusion transparente à l'idéologie soviétique pour laquelle le rouge avec ses nuances était une couleur pertinente. La représentation de l'ambiance vulgaire et gaillarde à la fois est complétée par la référence à Benia Krik, chef de gang et l'emblématique personnage des Récits d'Odessa d'Isaac Babel, constituant, à sa manière, une image stéréotypée d'Odessa connue de l'auteur et qui l'aide à accepter cette altérité quelque peu criarde et explicite.

L'immensité exceptionnelle des espaces en Russie soviétique semble incommoder l'auteur tout au long de son périple. C'est notamment le cas de Léningrad où cette exubérance spatiale semble se faire sentir le plus :

De l'autre côté de la Neva, des nuages obliques, rapides, font pleuvoir ombres et rayons sur le dôme de Saint-Isaac, la flèche de l'Amirauté, les bulbes du Sauveur-sur-le Sang, l'enfilade de 
façades nobles qui lie le jardin d'Été au palais d'Hiver : spectacle magnifique et dont la beauté, pourtant, comme l'avait senti Custine, est secrètement dégradée par l'excès d'espace. Rongée par le ciel et l'eau, segment d'un horizon, cette architecture monumentale a quelque chose de grêle, de relâché (pp. 30-31).

Dans l'exemple ci-dessus, nous croyons juste de noter l'antithèse des monuments emblématiques de Léningrad (dont l'énumération se résume à l'épithète appréciative " spectacle magnifique »), d'un côté, et de l'impression accablante qu'ils produisent. Cette impression se traduit par les métaphores « secrètement dégradée par l'excès d'espace » et « rongée par le ciel et l'eau », ainsi que par les épithètes " grêle » et " relâché ». Il est intéressant de noter la référence au marquis de Custine connu pour sa relation de voyage percutante sur la Russie du temps de Nicolas I ${ }^{\mathrm{er}}$ - comme cette référence n'est pas l'unique et que la justesse des jugements de Custine n'est jamais contestée à travers le texte de Rolin, on pourrait en déduire que, malgré le changement de régime politique, l'ambiance dans le pays, à son tour, n'a pas beaucoup changé, même un siècle et demi après, et que les mêmes stéréotypes restent toujours en vigueur.

Si l'excès d'espace sur les bords de la Neva n'est pas dépourvu d'un certain poétisme, l'image d'un hôtel à Yalta, au bord de la mer Noire, en est plutôt le contraire, frappant par son caractère rudement prosaïque :

L'hôtel Yalta ressemble à un gigantesque radiateur d'automobile : quinze étages, cent chambres par étage, aux balcons garnis d'ailettes de béton. De menus détails, vasistas arrachés de leurs charnières, commutateurs dévissés laissant apparaître un trou grossièrement foré dans le mur, robinets incontinents, plaques des auvents descellées, laissent imaginer la ruine pharaonique qu'il sera peut-être dans vingt ans, mais enfin, pour le moment, il ne faut pas être de mauvaise foi, c'est très laid, très moderne, très confortable [italiques de l'auteur cité] (Rolin, 1997, p. 64).

La description de l'hôtel est remarquable par la présence de deux épithètes traduisant l'idée de grandeur, à savoir " un gigantesque radiateur d'automobile » et « la ruine pharaonique ». Dans le contexte donné, ces épithètes décelent une attitude ironique de l'auteur, accentuée par l'accumulation « c'est très laid, très moderne, très confortable » où les deux dernières épithètes ont une valeur antiphrastique. Cette dernière serait parfaitement justifiée vu l'énumération des « commodités » de la chambre de l'auteur, ce qui met en valeur, une fois de plus, la priorité de la taille par rapport à l'aspect pratique que l'on observe si fréquemment en Union soviétique.

L'apothéose de la grandeur monumentale des bâtiments et des paysages soviétiques serait le centre-ville de Moscou, vu par Rolin en 1987. En voici la description :

Peu de paysages urbains aussi grandiosement sinistres que celui que l'on découvre du pont Kalinine à la tombée d'un jour d'hiver. Vers le couchant, la masse énorme de l'hôtel Ukraïna 
escalade le crépuscule jaune et noir, et l'on dirait que le peu de lumière rayonne de cette espèce de gouffre de pierre, Angkor Vat stalinien, navette spatiale de granit croisée de château de Chambord, urnes géantes, étoiles, faucilles et marteaux hérissés sur le ciel. Il est clair que les gens qui construisaient cela croyaient encore à quelque chose, à quoi, je ne sais pas, à la Russie, à la souffrance, à l'avenir radieux, enfin à quelque chose. De l'autre côté de la Moskova gelée, sur laquelle se sont abattus des vols de corbeaux, on aperçoit les gratte-ciel de la place de l'Insurrection et des Affaires étrangères, tours d'ombre avec des meurtrières de lumière pâle. [...] Moscou, avec son gigantisme froid, l'énormité étrange de ses monuments, semble une ville de Titans d'un autre âge, une cité d'un futur révolu et plutôt maléfique. Mais d'une force impressionnante [italiques de l'auteur cité] (Rolin, 1997, pp. 131-132).

Dans le présent exemple, nous croyons possible de dégager deux champs lexicaux majeurs : celui de la grandeur physique (" énorme », " gouffre », 《 géantes », « gigantisme », « énormité », «Titans ») et celui de la menace plus ou moins explicite ( sinistres », « le crépuscule », « tours d'ombre », « maléfique »), qui mettent en évidence le caractère peu accueillant, allant jusqu'au surnaturel, des espaces de Moscou. Pour mieux s'approprier cette altérité fascinante et inquiétante à la fois, l'auteur s'appuie sur les références culturelles qui lui sont déjà familières (et dont l'une appartient à sa culture d'origine), à savoir Angkor Vat et le château de Chambord. Nous noterons aussi l'oxymore « une cité d'un futur révolu » qui résume le caractère plutôt déplacé de la grandeur spatiale de Moscou, en l'occurrence, et des villes soviétiques dans leur ensemble.

Par ailleurs, ce paysage s'ouvrant depuis le pont Kalinine (qui s'appelle aujourd'hui « le pont Novoarbatski ») n'est pas le seul à surprendre l'auteur par son peu d'hospitalité et son caractère incommodant. Voici la description qu'il fait du Kremlin et du fameux Goum, centre commercial situé sur la place Rouge à Moscou :

Si les tours vert et rouge, les bulbes dorés, les créneaux bifides, les étoiles électriques brillant dans la nuit, les entassements irréguliers d'arches, d'ogives, de degrés de brique hérissés de noirs sapins, sous le vol des corbeaux, font du Kremlin «l'habitation qui convient aux personnages de l'Apocalypse» et qui impressionna si fort Custine, les trois carènes de verre renversées du Goum, de l'autre côté de la place Rouge, font de ce palais de l'Abondance un digne pendant architectural du palais de la Puissance, tout aussi dément que lui. L'Abondance a moins réussi en Russie que la Puissance, voilà tout (pp. 123-124).

Le caractère désordonné de l'architecture du Kremlin est accentué par une double énumération de ses éléments (« les tours vert et rouge, les bulbes dorés, les créneaux bifides, les étoiles électriques brillant dans la nuit, les entassements irréguliers d'arches, d'ogives, de degrés de brique hérissés de noirs sapins ») qui, aux yeux de l'auteur, ne sont pas vraiment faits pour être mis ensemble. Cet effet du désordre est aussi mis en valeur par le pléonasme " les entassements irréguliers » et définitivement résumé par l'épithète " dément ", cette dernière caractérisant le Kremlin au même titre que le Goum et traduisant l'extravagance 
excessive des deux bâtiments. Les nominations métaphoriques de ces deux endroits (" le palais de l'Abondance » et « le palais de la Puissance») soulignent leur caractère symbolique aux yeux de n'importe quel Soviétique, mais aussi leur valeur stéréotypisante pour la perception de Moscou par un voyageur étranger à l'époque. Toutefois, en définissant le Goum comme un " pendant architectural » du Kremlin (et faisant ainsi allusion à son infériorité par rapport à son voisin) et ayant recours aux métonymies "l'Abondance a moins réussi en Russie que la Puissance », l'auteur conclut, une fois de plus, que le confort matériel est fréquemment négligé en U.R.S.S. en faveur de la monumentalité apparente.

\section{Les déceptions et les imprévus spatioculturels}

Paradoxalement, la taille de certains bâtiments emblématiques de l'U.R.S.S. est près de décevoir l'auteur, faute de grandeur attendue. Tel est le cas du mausolée de Lénine qui ne semble que très peu impressionner Rolin :

Devant le mausolée de Lénine, dont la taille surprend par sa modestie et, à dire vrai, les formes trapues, couleur de sang caillé, par leur intégration à l'ensemble du Kremlin, un milicien qui joue avec sa matraque, qui a l'air de se considérer comme personnellement propriétaire de l'édifice, me regarde droit dans les yeux, sans proférer un son, lorsque je lui demande à quelle heure les visites sont autorisées. Peut-être la dernière demeure de Vladimir Ilitch est-elle, à la façon d'un sérail, gardée par des muets ? (Rolin, 1997, p. 121).

Le paradoxe « dont la taille surprend par sa modestie » traduit les attentes trompées de l'auteur face à la vision stéréotypée de la grandeur des espaces russes et soviétiques. Nous sommes également en présence d'une certaine ironie de l'auteur rendue par le biais de la comparaison du mausolée à un sérail. Dans le même temps, l'épithète « couleur de sang caillé » nous paraît pertinente, car elle nous renvoie, de manière implicite, à l'époque sanglante que fut la révolution de 1917 (dont Lénine avait été l'un des principaux instigateurs) aussi bien que les décennies qui suivirent.

Une autre déception guette l'auteur à Yalta, lors de la visite du célèbre palais de Livadia : «Le palais de Livadia est blanc, pas très beau, pas très grand, lourdaud, genre grosse villa d'Antibes à pergola sur le toit» (p. 76). Les litotes « pas très beau » et " pas très grand », de même que les épithètes « lourdaud » et « grosse villa» traduisent le manque d'élégance du palais en question. Encore une fois, nous sommes en présence d'une référence culturelle appartenant à la culture d'origine de l'auteur, qui sert, manifestement, à lui faire mieux vivre son désenchantement.

Parmi les références culturelles russes, on en trouve aussi qui aident l'auteur à maîtriser une altérité jusqu-là inimaginable. Nous pouvons citer, en guise d'exemple, la description de certains cafés de Leningrad : 
m'avaient toujours paru formules toutes faites et sans véritable référent dans la réalité olfactive, sur les bistrots « empuantis », l'air « empesté » qui stagne dans les lieux où se perd le pauvre Marmeladov [guillemets de l'auteur cité] (p. 39).

Les épithètes à valeur négative " les bistrots 'empuantis'» et «l'air 'empesté' », aussi bien que la mention de Sémion Marmeladov, personnage dostoïevskien devenu symbole de déchéance, nous laissent évaluer toute l'agression olfactive des établissements en question. Une autre réalité culturelle, à savoir la queue (partie intégrante du quotidien de tous les Soviétiques), est aussi citée par l'auteur afin de démontrer la justesse des deux stéréotypes qu'il doit vérifier de manière empirique.

Une autre référence culturelle russe qui amortit l'effet d'imprévu éprouvé par l'auteur face à certaines manifestations de l'altérité culturelle, est l'œuvre d'Anton Tchékhov. En voici un exemple :

Le défraîchi, le légèrement délabré, qualités dont la Russie n'est pas avare, siéent au genre balnéaire : les couleurs fanées, bleu ou terre de Sienne, des milliers de lits lattés couvrant le rivage, le bois salé et friable de l'espèce de petite gare, à véranda et clocheton vitré, où siègent les maîtres nageurs, donnent à la plage des Komsomols un charme qu'on osera dire tchékhovien (p. 54).

Les lexèmes « le défraîchi » et " le délabré » exprimant l'état plutôt dégradé d'une chose, accompagnés de la litote « qualités dont la Russie n'est pas avare », évoquent l'abandon de cette plage d'Odessa. Dans le même temps, la mention de Tchékhov à côté du verbe « oser » traduit le caractère quelque peu exagéré de cette comparaison, pourtant celle-ci paraît indispensable à l'auteur pour mieux s'approprier l'altérité qu'il est en train de découvrir.

\section{Des réalités spatioculturelles familières}

Toutefois, l'exploration des espaces soviétiques par Olivier Rolin n'est pas complètement dépourvue d'impressions positives. Nous noterons, à ce titre, la description des rues d'Odessa qu'il fait ci-dessous :

De toutes les villes quej'ai vues en Union Soviétique, celle où le père Goriot rêvait d'aller fabriquer des pâtes est certainement la plus plaisante, la plus marquée par son ancien cosmopolitisme. De larges rues à gros pavés dodus, bordées d'immeubles ornés, peints, volontiers excessifs dans leur décoration, « prétentieux » diraient ceux qui ignorent la nostalgie, inclinent vers le port. Un air de Gênes ou de Nice. Ici, des restaurants, des cafés presque agréables, des terrasses! Où on peut espérer s'asseoir! Ici, des étals en plein vent, brillants de petites pommes vertes, de petits oeufs très blancs, de tomates, de poivrons! Des grands arbres, des jardins publics, le murmure des fontaines, le roucoulement des tourterelles! [guillemets de l'auteur cité] (Rolin, 1997, p. 45).

Dans cet exemple, nous voyons l'auteur s'appuyer sur trois références culturelles européennes, dont deux françaises (la ville de Nice et le célèbre 
personnage balzacien), qui lui sont manifestement bien familières. La périphrase « celle où le père Goriot rêvait d'aller fabriquer des pâtes » servant à nommer Odessa, démontre la compréhensibilité de cette ville pour l'auteur, du moins en comparaison avec Léningrad qu'il venait de quitter et où il avait rencontré de nombreuses difficultés à la recherche d'un endroit où manger le soir (d'où l'exclamation « Où on peut espérer s'asseoir! »), les personnes seules n'y étant pas les bienvenues dans les restaurants. Les énumérations des éléments constituant les paysages habituels des villes européennes (notamment celles du Sud) nous font comprendre que l'effet d'altérité culturelle est, dans le cas donné, quasiment nivelé. La seule chose qui révèle sa présence, est l'épithète " des cafés presque agréables ", où l'adverbe " presque » désigne le degré de perfection qui n'est pas encore atteint. Le nombre important d'exclamations dans le fragment cité est censé traduire la forte impression positive que l'auteur a de ce paysage urbain.

Nous allons aussi noter une heureuse exception parmi les cafés « empuantis » de Leningrad cités avant :

Le Literatournoïe Kafe, perspective Nevski, est un des lieux où se préserve quelque chose du raffinement ancien de Pétersbourg. Peu de tables, sous des voûtes blanches, éclairées par des luminaires en forme d'arbustes dorés, portant des oiseaux de verre. Un violon, un violoncelle accompagnent une chanteuse en robe noire. Pouchkine, dit-on, vint ici au petit matin du 27 janvier 1837 avant de se rendre à la Rivière Noire où il allait être mortellement blessé [italiques de l' auteur cité] (p. 109).

Il est intéressant de constater que c'est l'image de l'ancienne Saint-Pétersbourg qui possède une connotation manifestement méliorative par rapport à celle de la Léningrad soviétique. Les éléments du décor énumérés dans le passage ci-dessus, qui évoquent le "raffinement ancien ", et surtout la référence à Pouchkine en tant que figure emblématique de l'âge d'or de la culture classique russe, ne font que souligner l'effet d'évasion instantanée assuré par ce vestige de l'ancienne capitale de l'Empire russe que l'auteur ne manque pas d'apprécier malgré son ancien engagement politique révolutionnaire.

\section{Espace à usage inattendu}

Pour terminer notre analyse, nous allons évoquer un endroit à fonction détournée, tout particulier aux yeux de l'auteur, mais parfaitement ordinaire en Union soviétique. Il s'agit d'une église transformée en station téléphonique, et en voici la description faite par Olivier Rolin (1997):

Il y a, rue Ogareva, non loin du Kremlin, une église qui n'a sûrement pas sa pareille dans le monde. Tout le long de la nef, comme autant de chapelles, sous des vitraux modernes, sont disposés des cabines téléphoniques et les tableaux des codes interurbains. On entend hurler une paysanne qui doit avoir une conversation avec Vladivostok. En haut des quelques marches qui menaient à l'autel, trois guichets ont été pratiqués dans un rideau de bois ondulé qui masque 
et révèle ainsi les préposées exactement comme l'ancienne iconostase, dont il occupe la place, masquait et révélait l'officiant. Les saints orthodoxes ont été remplacés par des allégories ailées des télécommunications (pp. 137-138).

Dans l'exemple ci-dessus, l'auteur dresse de nombreux parallèles entre les deux fonctions de cette église (celle d'avant et celle du moment présent), en comparant les cabines téléphoniques aux chapelles, les préposées aux officiants, les images des saints aux allégories des télécommunications. Cependant, c'est le verbe « hurler » qui saute aux yeux du lecteur et qui rompt la prétendue continuité, puisque les hurlements, chose difficilement concevable dans une église dans sa fonction première, semblent habituels dans une station téléphonique où les clients devaient élever la voix, d'abord, en raison de la mauvaise connexion et, en plus, suite à l'idée reçue, fréquente en U.R.S.S., que plus fort on parlait en téléphonant, mieux on se faisait entendre, surtout si l'interlocuteur se trouvait dans une ville éloignée. C'est surtout par le biais de ce verbe que l'auteur semble exprimer son choc face à cet exemple d'altérité jamais vue jusque-là.

\section{Conclusion}

En dressant le bilan, nous croyons juste de noter que la découverte des espaces de l'Union soviétique par Olivier Rolin, qu'il relate dans son récit de voyage En Russie, se révèle pour lui une véritable expérience de l'altérité culturelle. Parmi les principaux procédés linguistiques servant à traduire cette altérité spatiale de l'U.R.S.S. vis-à-vis des attentes de l'auteur, nous croyons pertinent de citer des épithètes à valeur méliorative ou péjorative, des hyperboles, des antithèses, des antiphrases qui appuient, le plus souvent, un stéréotype ou une référence culturelle à base de la description d'un lieu. Le cas particulier est représenté par une église moscovite à fonction détournée, réaménagée en station téléphonique où l'on ne trouve pas de parallèles culturels franco-russes, mais où toute la dissonance éprouvée par l'auteur est traduite par le verbe « hurler » qui exprime, à lui seul, la dégradation de l'ambiance du lieu à la suite de cette transformation effectuée par le régime communiste connu pour sa politique antireligieuse (Pierre, 1961).

Il est à noter que le stéréotype majeur concernant l'espace russe et soviétique, est celui de sa grandeur physique au nom de laquelle on sacrifiait souvent l'aspect pratique des lieux et qui déçoit explicitement l'auteur en raison de son insuffisance et de son peu de confort (contrairement aux attentes qu'il avait eues), ou se montre excessive et, par conséquent, peu commode, voire effrayante.C'est le cas de plusieurs monuments emblématiques de l'Union soviétique, tels que le Kremlin, le mausolée de Lénine, le palais de Livadia. En revanche, certains endroits qui ne sont pas lestés de dimensions excessives et qui gardent le charme d'avant révolution (tels que le Literatournoïe Kafe à Leningrad) sont représentés de manière méliorative et à travers les références culturelles russes à connotation 
manifestement positive. Par ailleurs, les lieux qui ont de la ressemblance avec des villes familières à l'auteur et où l'altérité ne se fait pas tellement sentir, sont décrites en se fondant sur des références culturelles françaises et européennes, plus proches de l'auteur et aussi de son lectorat français. Ainsi, sa perception de cette altérité est considérablement déterminée par les stéréotypes et les références culturelles françaises et russes (concernant ces dernières, l'auteur en démontre une connaissance profonde, ce qui témoigne du fait qu'il était bien préparé à ce voyage), mais aussi internationales, qui, dans certains cas, s'avèrent bien fondés, tout en se déconstruisant dans d'autres cas, face à la réalité.

\section{References}

Amossy, R. (1989). La notion de stéréotype dans la réflexion contemporaine. Littérature. Mutations d'images, 73, 29-46. Retrieved February 1, 2020, from https://www.persee.fr/doc/litt.

Garrec, N. (2011). Images de l'Union soviétique dans la presse française des années trente : rapport entre texte et image. Lyon: Université Lumière Lyon 2.

Goldman, E. (2019, 16 September). Qu'est-ce que les touristes étrangers étaient autorisés à voir en URSS ? Russia beyond.. Retrieved March 14, 2020, from https://fr.rbth.com/ histoire/83525-touristes-etrangers-urss.

Lamarre, M. (2014). Ruines de l'utopie. Antoine Volodine, Olivier Rolin. Villeneuve d'Ascq: Presses universitaires du Septentrion.

Mendras, M. (1985). La France dans la politique occidentale de l'URSS. Politique étrangère, 3, 653-668. Retrieved July 14, 2020, from https://www.persee.fr/doc/polit_0032-342x.

Pierre, A. (1961, April). La vie religieuse en Union soviétique. Le Monde diplomatique. Retrieved March 17, 2020, from https://www.monde-diplomatique.fr/1961/04/PIERRE/24202.

Rolin, O. (1997). En Russie. Paris: Éditions du Seuil. 